\title{
An Evaluation Technique for Content Interaction in Web-based Teaching and Learning Environments
}

\author{
Xu Lei, Claus Pahl, Dave Donnellan \\ Dublin City University, Computer Applications, Dublin 9, Ireland
}

\begin{abstract}
Interactivity is a central element in teaching and learning. The Web as a new educational platform enables new forms of teaching and learning. The consequence for the Web - a constantly evolving environment that provides less direct feedback - is the importance of evaluation. In particular the learners' interaction with the Web-based multimedia features is central. We propose Web usage mining, a technology that has already been used to analyse e-commerce Web sites, in conjunction with an analytic model as the evaluation approach.
\end{abstract}

\section{Motivation}

Interaction is central in teaching and learning. The learning process is based on interactions with the instructor, with peers and with content. Recent Web and multimedia technologies provide a wide range of possibilities for learners to interact with content and also other learners and instructors [4]. The Web as the platform, however, causes problems for two reasons. Firstly, the teacher receives less direct feedback. Secondly, it enables new and innovative forms of teaching and learning. Formative evaluation and the reflection of evaluation results in the instructional design are therefore paramount in this context.

We propose Web usage mining [1] as an analysis technique. It aims at the discovery and extraction of behaviour patterns and rules from user access logs created by a Web server. Access logs reflect user activities. Web usage mining is widely used in e-commerce systems as a means to analyse customer shopping behaviour. It can be of equal importance to the educational context, helping to improve instructional design and implementation of Webbased teaching and learning systems [5].

We are concerned with the analysis and interpretation of in particular the learners' interaction with content - the Web is per se a content-oriented presentation medium. Knowledge-level content interaction of the learner with features and media of a course Web site is therefore a central instructional design and evaluation aspect that crucially impacts the quality of interaction and learning overall. Monitoring and understanding learner behaviour is a key motivation. Analysing behaviour, i.e. discovering, extracting, and representing learner behaviour in terms of basic activities, is the first step in the evaluation process. The second step concerns the interpretation of the analysis results in the educational context. An explicit or implicit analytic model provides the cognitive and pedagogical context in which this interpretation can take place.

\section{Web Mining for Educational Systems}

Web access logs are a reflection of the user's activities and interactions in the system. The key problem of Web mining is to reduce the amount of data and to filter out essential and meaningful information from a full Web $\log$. A variety of learning-related information can be extracted from Web logs. Individual activities are usually part of larger and more comprehensive learning tasks, whose implementation by the learner is governed by the learner's objectives and learning strategies.

The determination of the actual learner activity is difficult, but can be derived from the expected (and supported) activity on a page and the time spent on that page. Another aspect relates to the ordering of learning elements, i.e. the sequencing of elements by the learner has to be taken into account. Individual behaviour is abstracted into patterns. A comparison technique compares expected and actual behaviour patterns.

We have developed a session classification technique to identify the main purpose(s) of a learning session. A learning session is a period of uninterrupted work with the system. Determining the main session activities identifies the most likely learning task and objective.

Time series can be used to detect changes in behaviour. A learning strategy is often based on a learner's focus on different aspects during a course. Learning objectives differ, e.g. between term and exam preparation periods. 


\section{Evaluation of Web-based Active Learning}

Our primary objective is the explanatory function of Web mining, which allows us to understand learners better and to convert this understanding in a formative evaluation process into improved instructional designs. We have applied our techniques in an integrated virtual course environment for $3^{\text {rd }}$-level computing students that provides a range of active learning features [3]. Using education-specific Web mining techniques has resulted in confirmation of instructional design decisions and also in significant quality improvements.

Expected (and supported) behaviour does not always coincide with the actual behaviour of learners. The actual behaviour is not always obvious and easy to extract. An analytic model providing a language to express and compare behaviour patterns - expected and actual ones is particularly important here. A sequential pattern analysis yields sequences of actual learning activities, called sequential patterns. An expected behaviour pattern is expressed in terms of a richer, more abstract language adding concepts such as repetition, choice, and parallel usage to sequences of activities. A comparison analysis provides important feedback for the instructor.

One part of the Web-based course environment is a tutorial-style active learning feature based on a guidedtour idea. Students are supposed to work interactively with an online tool through a sequence of separate lessons. Using our comparison technique we found that actually $84 \%$ of all student learning sessions followed the expected pattern formulated by the instructor. In this case Web mining confirmed the successful implementation of this highly interactive feature.

Often, novel and innovative features are added to an existing environment. In this case, an in-depth evaluation of these features is required. In the course environment under consideration, at one stage scaffolding features were included in the Web content in order to make the system more suitable for autonomous, self-reliant learning. The idea behind scaffolding features is that during the course the learner becomes more self-reliant with the help of a coach, active work, and reflection. A reduced provision of online help for later units of the course should therefore be possible and a reduced usage of these scaffolding features during the course of time could therefore be expected. A mining-based evaluation of usage patterns showed that the actual usage of scaffolds for later lessons in the course material had reduced to around $35 \%$ of the original number. Again, this constituted a confirmation of the approach in general. However, some improvements - related to level and quality of coaching - were made based on the patterns that were discovered.

We have already discussed both learning strategy detection and session purposes as change indicators.
Constant monitoring of student behaviour through these high-level indicators of had been an important feedback element for the instructor during the course delivery time.

\section{Discussion and Conclusions}

The novelty of the Web as an educational platform and the consequent lack of experience and best practice require a thorough design and in-depth analysis of the learner's behaviour in this new infrastructure. Our focus in this investigation has been on the learner interaction with content. Education-specific Web usage mining is an ideal tool to support learner behaviour analysis. A formative evaluation based on this technique can lead to improved instructional designs. Web mining is essentially an observation-oriented analysis method. This method has several advantages over classical observation approaches. Web mining is objective, non-interruptive, automatable, and always available. Web mining should, of course, be complemented by other evaluation techniques such as surveys and, if available, an analysis of student attainment and learner profiles.

Web mining for educational systems is a powerful analysis tool, in particular for active learning oriented environments [3]. Nonetheless, a critical aspect in the evaluation process is the interpretation of the mining results within the application domain. An analytic model provides the necessary context for this interpretation. Central in the learning process are the knowledge-level interactions of a learner with the content in particular. Only if we manage to integrate pedagogical and technical concepts of activity and form a coherent analytic model, we are able to successfully improve instructional design for Web-based courses through Web and data mining.

\section{References}

[1] Chang, G., Healey, M.J., McHugh, J.A.M., and Wang, J.T.L. Mining the World Wide Web - An Information Search Approach. Kluwer Academic Publishers. 2001.

[2] Nardi, B. (ed.) Educational Context and Consciousness: Activity Theory and Human-Computer Interaction. MIT Press. 1997.

[3] Pahl, C. Interactivity and Integration in Virtual Courses. Proc. International Conference on Advanced Learning Technologies ICALT 2001. IEEE. 2001.

[4] Weston, T.J. and Barker, L. Designing, Implementing, and Evaluating Web-Based Learning Modules for University Students. Educational Technology, 41(4):15-22. 2001.

[5] Zaiane, O.R., and Luo, J. Towards Evaluating Learners' Behaviour in a Web-Based Distance Learning Environment. Proc. International Conference on Advanced Learning Technologies ICALT'01. IEEE. 2001. 